\title{
La géographie des ambassades et des consulats en Allemagne et l'effacement de l'ancienne frontière allemande (1984-2014)
}

The geography of embassies and consulates in Germany and the blurring of the former inner-German border (1984-2014)

Die Verteilung der Botschaften und Konsulaten in Deutschland und die Löschung der ehemalige deutsch-deutsche Grenze (1984-2014)

\section{Antoine Laporte}

\section{OpenEdition}

\section{Journals}

Electronic version

URL: http://journals.openedition.org/rge/5872

ISSN: $2108-6478$

Publisher

Association des géographes de l'Est

\section{Printed version}

Date of publication: 1 December 2016

ISSN: 0035-3213

\section{Electronic reference}

Antoine Laporte, « La géographie des ambassades et des consulats en Allemagne et l'effacement de l'ancienne frontière allemande (1984-2014) ", Revue Géographique de l'Est [Online], vol. 56 / n³-4 | 2016, Online since 30 December 2016, connection on 08 September 2020. URL : http:// journals.openedition.org/rge/5872

This text was automatically generated on 8 September 2020 .

Tous droits réservés 


\title{
La géographie des ambassades et des consulats en Allemagne et l'effacement de l'ancienne frontière allemande (1984-2014)
}

\author{
The geography of embassies and consulates in Germany and the blurring of the \\ former inner-German border (1984-2014) \\ Die Verteilung der Botschaften und Konsulaten in Deutschland und die \\ Löschung der ehemalige deutsch-deutsche Grenze (1984-2014)
}

Antoine Laporte

1 Il est indéniable que le maintien de disparités, notamment économiques, entre les deux anciens périmètres de l'Allemagne durant la Guerre froide, constitue une clé de lecture incontournable du territoire allemand, même encore un quart de siècle après la Chute du mur de Berlin. Pourtant, la géographie régionale dans ce pays ne peut en aucun cas s'y réduire. L'ancienne division entre un « est » et un « ouest » semble très structurante en ce qui concerne le chômage ou la production industrielle, mais l'est en revanche beaucoup moins en démographie, pour ce qui concerne les soldes migratoires ou les taux de fécondité (Laporte, 2014). Il existe ainsi de très nombreux domaines qui permettent d'observer aussi bien le maintien de disparités que des convergences dans les conditions économiques et sociales entre les deux parties de l'Allemagne. Structurant sans être absolu, le rôle de l'ancienne frontière interallemande permet de comprendre un certain nombre de logiques inégalitaires dans le pays. Cependant, le risque serait de donner une trop grande importance au rôle de l'ancienne frontière interallemande, voire de la considérer comme discontinuité a priori. Une lecture fine des fractures du territoire allemand fait souvent apparaître des structures bien plus complexes. Cet article propose une analyse visant une perspective déductive et diachronique des discontinuités du territoire allemand, afin de tester l'hypothèse d'une limite qui s'estomperait entre anciens et nouveaux Länder à partir des aires d'influence et de compétence des villes. Ces dernières sont évidemment le fruit de critères 
nombreux et pour la plupart de nature économique. Pour ma part, je tente de les mettre en évidence avec la répartition des ambassades et des consulats dans le pays.

2 La géographie diplomatique, entendue dans cet article comme l'étude des logiques de localisation des ambassades et des consulats est un domaine sinon émergent, du moins relativement confidentiel en géographie. Le plus souvent, ces travaux se portent sur des échelles très petites, en général mondiales (Vogeler, 1995; Neumayer, 2008; Beauguitte et Didelon, 2011). Le corps diplomatique y est étudié pour ce qu'il est en premier lieu, à savoir le révélateur direct des aires d'influence en matière de politique étrangère d'un Etat. Sa géographie conduit plus ou moins à produire un tableau à un moment donné des relations internationales. Il arrive à l'inverse que l'intérêt pour la géographie diplomatique interroge des échelles très locales, avec la question des implantations d'ambassades (Fischer et alii, 1998 ; Fleischmann, 2005 ; Mamadouh et alii, 2015). L'espace considéré, par son architecture ou sa position de la ville, est également analysé comme un vecteur de la puissance d'un Etat et de sa mise en scène. Dans cet article, je m'intéresse à la géographie diplomatique, définie par l'étude de la répartition des institutions à vocation diplomatique, ici les ambassades et les consulats sur le territoire national d'un Etat de 80 millions d'habitants. Même si, rapportés à l'ensemble des services publics, ces ambassades et ces consulats n'ont qu'un impact négligeable en nombre de salariés ou en capacité à influer sur les pratiques de mobilité et de résidence de la population allemande comme les étrangers vivant en Allemagne, je fais l'hypothèse que leur localisation et les aires de compétence de ces établissements sont révélateurs des logiques en terme de hiérarchie urbaine.

3 La géographie des ambassades et des consulats permet d'aborder la question des frontières intérieures de l'Allemagne par l'observation de la manière dont des acteurs extérieurs au pays posent des choix de localisation, entre Bonn et Berlin pour les ambassades et entre les autres villes allemandes pour les consulats. En ce qui concerne ces derniers, les Etats représentés sont poussés à définir l'étendue des compétences territoriales de chacun d'eux. Les ambassades et les consulats à la manière de nombreux services publics mais à la différence de la plupart des services marchands ne dessinent pas une aire de chalandise, mais des territoires sur lesquels s'étendent leurs compétences, désignés dans le cas étudié ici par le terme de "circonscriptions consulaires » (CC). En ce qui concerne l'Allemagne, la géographie des établissements diplomatiques interroge donc à la fois l'évolution du système de villes mais aussi des territoires sur lesquels elles jouent un rôle de chef-lieu.

Comment deux systèmes diplomatiques, celui abrité par l'Allemagne fédérale et celui de la République Démocratique Allemande (RDA) n'en ont formé qu'un seul ? La discontinuité héritée de la frontière interallemande, considérée a priori comme une ligne de lecture du territoire allemand, reste-t-elle pertinente à l'aune de la réorganisation des sphères d'influence des villes? Ou observe-t-on l'émergence durant cette période d'autres lignes de fracture? Au-delà d'une simple question sur les découpages territoriaux, la problématique intègre évidemment une réflexion sur la manière de considérer les processus territoriaux postérieurs à la Réunification. Un premier moment de cet article cherche à présenter les enjeux d'une recherche spécifique sur la pérennité d'une lecture très marquée par le découpage est-ouest hérité de la Guerre Froide avant de présenter la répartition des ambassades et des consulats sur le territoire allemand. Enfin, je me pencherai sur la géographie des circonscriptions consulaires. 


\section{La nécessité d'un renouvellement de la lecture de l'espace allemand}

5 L'étude de l'Allemagne, en particulier dans la géographie française, semble souvent déterminée par le primat de la division est-ouest, qui se doit, un quart de siècle après la Réunification d'être non pas réfuté mais discuté et relativisé.

\section{A. De la pertinence du maintien de la limite est-ouest en géographie régionale}

6 Le découpage du territoire a priori est un exercice intellectuel fréquent en géographie régionale, lorsqu'on cherche à rendre intelligible un espace divers. Il s'agit d'opérer une catégorisation des espaces, un peu comme dans d'autres domaines des sciences sociales, on essaie de le faire avec des groupes sociaux, des biens ou des catégories intellectuelles. La Réunification a eu de tels effets sur l'espace allemand que les études en géographie sur l'Allemagne des années 1990 et 2000 en ont été mécaniquement très marquées. Les manuels de géographie régionale de l'Allemagne parus peu après la Chute du mur font de la Réunification et de l'unité nationale leur problématique (Lebeau, 1991 ; Jones, 1994). Des ouvrages plus spécialisés, en particulier en géographie économique ou industrielle ont également abordé la question (Carroué et Odent, 1994). Un des seuls textes à proposer en soi une régionalisation systématique de l'Allemagne comme cadre d'analyse et à ne pas reprendre le découpage est-ouest est celui de Pierre Riquet dans la Géographie Universelle, dont le tome consacré à l' "Europe médiane ". Jusqu'au milieu des années 2000, il semble être plus l'exception que la règle. La Réunification de l'Allemagne et ses conséquences sur Berlin et les nouveaux Länder ont également constitué la toile de fond économique, culturelle et sociale aux travaux portant sur ce pays, voire parfois le cadre d'analyse de la géographie générale de l'Allemagne, en particulier en France. En effet, la restructuration des espaces berlinois a été abordée à de nombreuses reprises, qu'il s'agisse de son statut renouvelé de capitale culturelle (Grésillon 2002; Krätke 2002; Boichot 2012), de son accession ou non dans le club fermé des villes globales (Cochrane et Passmore 2001; Maccarone et Sbordone 2003) ou de sa métamorphose en lieu d'exercice du pouvoir (Laporte 2016a). Les autres régions de RDA ont beaucoup attiré l'attention, que ce soit sous l'angle de l'émergence de l'échelon régional (Sur 1997; Queva 2007) de la désindustrialisation (Von Hirschhausen et Roth 2003; Roth 2008), de la décollectivisation des terres agricoles (Hirschhausen et Lacquement (eds.) 2007; Vonau et al. 2012), ou encore au sujet de l'héritage du tracé de l'ancienne frontière interallemande (Charlot 2008).

$7 \quad$ L'intérêt apparemment plus grand pour les nouveaux Länder s'explique encore une fois aisément par l'impact inédit des phénomènes de transition post-socialiste à savoir la modification de territoires façonnés par une économie planifiée par une économie de marché et des régimes politiques démocratiques. En Allemagne, la Réunification construit une situation inédite en Europe de confrontation de deux héritages socioéconomiques et législatifs à l'origine de disparités régionales sur un même territoire national. Cependant, malgré cela, l'étude récurrente de ces phénomènes peut avoir un possible effet pervers, celui de considérer l'ancienne frontière interallemande, qui 
recoupe toujours des limites entre Länder, comme une limite intérieure majeure a priori inhérente à toute description de l'espace allemand. Le risque est d'observer des phénomènes d'ordre spatial par un miroir déformant. Par exemple, le PIB par habitant, s'il est en moyenne plus faible à l'est qu'à l'ouest, ne permet pas de tracer une ligne de discontinuité suivant exactement l'ancienne frontière interallemande. Alors que $39 \%$ des chômeurs allemands étaient Ossis en 2001, leur part n'est plus que de 27\% en 2015. Certaines parties de la Basse-Saxe ont des niveaux de production de richesse proches de la région voisine anciennement est-allemande de Saxe-Anhalt (Commission européenne 2014; Eurostat 2015). Berlin, toujours déclassé par rapport aux autres grandes villes, présente en matière de PIB par habitant ou de pouvoir d'achat des niveaux supérieurs à la plupart des régions rurales, à l'est comme à l'ouest. De même, si le chômage est de 4 points supérieur dans l'ancien périmètre fédéral par rapport aux nouveaux Länder, la différence est également très nette, toutes choses égales par ailleurs, entre espacesruraux et urbains.

8 La question sous-jacente à ces réflexions sur la pérennité de la mesure de la Réunification concerne la temporalité au-delà de laquelle il semble impropre de ne parler que de post-socialisme pour comprendre les phénomènes spatiaux consécutifs à la fin de la Guerre Froide en Europe Centrale. Nous sommes en 2015 aussi éloignés de la Chute du Mur que nous l'étions en 1971 de la chute du nazisme, époque qu'on ne désigne plus $d$ ' "après-guerre ». $18 \%$ de la population allemande est née après la Réunification. Les plus jeunes ont pour la plupart des parents qui n'étaient qu'enfants en 1989. Le contexte économique en Europe, après la crise de 2007, est aujourd'hui bien différent de celui du début des années 1990. L’Allemagne dépeinte alors comme un colosse aux pieds d'argile a connu au début des années 2010, une croissance bien supérieure à nombre de ses voisins. Deuxième argument lié à la temporalité de la Guerre froide, certaines disparités actuelles préexistaient déjà avant 1945. À Berlin, au $\mathrm{XIX}^{\mathrm{e}}$ siècle déjà, les arrondissements de l'ouest de la ville étaient plus huppés que les quartiers ouvriers à l'est et leurs Mietskasernen.

$9 \quad$ L'Allemagne est-elle donc sortie du post-socialisme ? La question n'est pas aisée parce qu'elle demande de définir précisément les indicateurs du marquage de l'espace par le socialisme et ses conséquences. La Wende, terme que les Allemands utilisent pour parler des changements politiques de l'époque de la Réunification, renvoie beaucoup plus à un événement ou à une suite d'événements rapprochés dans le temps, qu'à une période. Dans le droit, et surtout dans le droit du travail, l'ancienne RDA n'a plus de régime d'exception. La division a été figée dans les statistiques. L'institut fédéral de statistique Destatis a d'ailleurs gardé l'habitude de fréquemment séparer dans ses tableaux les «nouveaux Länder » et l' " ancien périmètre fédéral» (früheres Bundesgebiet). Le terme de résilience, fort employé de nos jours, a été utilisé également dans le domaine de la transition post-socialiste. Apparemment approprié à la question de la fin d'une transition, il n'est pas aisé à manier du fait de la difficulté à établir les caractères d'un retour à un état stable des territoires actuels et il contribue surtout à penser la période de la Guerre froide comme une parenthèse et moins comme un passage qui peut s'avérer plus ou moins structurant dans l'espace que la période qui lui a succédé, dans laquelle domine une économie libérale.

10 L'incertitude quant au vocabulaire adéquat pour décrire la géographie actuelle impose donc de chercher des indicateurs ou des éléments pouvant servir de points de repère. La répartition des services publics constitue également un marqueur intéressant de 
l'évolution des disparités territoriales en Allemagne. Leur maillage actuel est l'héritier de la période de la division comme de celle de la Réunification. Les fermetures de crèches, généralisées à l'est, font partie des images d'Epinal de la violence et de la rapidité des processus de transition dans les années 1990. Le phénomène a été renforcé parce que les services publics en Allemagne sont beaucoup pris en charge par les Länder et de plus, la division entre est et ouest s'est perpétuée pendant une vingtaine d'années dans les salaires des fonctionnaires et le droit du travail en général. Le phénomène d'adaptation des services publics après 1990 est complexe. Elle ne répond ni à une simple apposition des canons ouest-allemands des services publics sur les régions orientales, ni à une rapide déliquescence de l'héritage d'un appareil d'Etat sans moyens. Leur géographie actuelle dépend de plusieurs grandes logiques. Tout d'abord, pour ce qui est de l'administration fédérale. La Réunification s'accompagne d'une révision de la géographie de l'appareil d'Etat avec des services déconcentrés qui s'implantent, avec leur cortège d'emplois, dans l'ensemble des régions d'ex-RDA en parallèle de mouvements de transferts d'institutions de Bonn à Berlin(Laporte 2016). Ce phénomène conduit donc à un rééquilibrage mais dans un domaine peu pourvoyeur d'emplois. La substitution d'entreprises industrielles par des administrations de haut niveau n'a eu que des effets marginaux et n'a en rien enrayé les difficultés économiques de la fin des années 2000. Plus importantes ont été les conséquences de l'adaptation du réseau de services publics à la conséquence conjointe d'un solde migratoire très négatif et d'un effondrement de la fécondité, et donc à une baisse de la population. Le phénomène a aussi été décrit dans le domaine des réseaux d'approvisionnement en eau potable et de l'adaptation des infrastructures à une réduction très importante de la demande (Huning et al. 2011; Florentin 2015). Le déclin de la population a aussi entraîné (ou a été accompagné par) une réduction du nombre des structures dans le domaine de l'éducation (Barakat 2015) ou de la santé. Dans ces derniers secteurs, la question est autant la résultante d'une baisse de la population, que le moyen de faire face à des réductions budgétaires (Hentschker, Mennicken et Schmid 2014).. Le pendant de ces processus est présenté récemment sous les traits d'une " périphérisation » (Kühn 2014) des espaces ruraux allemands, indépendamment du côté du rideau de fer dans lesquels ils se trouvaient. Dans de nombreux travaux, la Réunification apparaît comme un élément de contexte indépassable même si dans les années récentes, la métropolisation ou les écarts entre grandes villes et zones rurales sont davantage mis en avant.

\section{B. La diplomatie, un service public marginal mais au marquage spatial révélateur}

11 La diplomatie se présente comme un cas de "service rare " au sens où l'entend Claude Manzagol (1991) à savoir un service peu présent sur un territoire, avec peu d'employés et d'usagers, et qui donc imprime une aire de compétence ou d'influence étendue. Les choix de localisation des établissements dans ce domaine sont d'autant plus réfléchis que ces structures coûtent cher aux chancelleries. Le thème du marquage spatial de la diplomatie est apparu par le biais d'études en relations internationales. La géographie s'est emparée de la question par une recherche de quantification des relations entre Etats (Brams, 1966 ; Beauguitte et Didelon 2011). Elle part du constat que sur l'ensemble des relations bilatérales possibles entre les presque 200 Etats de la planète, seules $30 \%$ seulement se concrétisent par un échange d'ambassades (Neumayer, 2008). Ainsi, par 
exemple, Eric Neumayer propose une étude des facteurs de choix dans la représentation des Etats en fonction de variables comme la proximité, les compatibilités idéologiques et les relations économiques. Les auteurs de ces études montrent un élément intéressant, à savoir que le tissu des structures diplomatiques peut être abordé comme un élément quantifiable (et donc objectivement comparable) de la puissance. L'intensité de la présence d'ambassades a pu être étudiée de différentes manières, mettant à jour des structures dans la capacité des Etats à s'investir selon les parties du monde. Ces travaux, assez éloquents pour différencier les Etats puissants et influents d'une part, et les «diplomaties faibles » (Beauguitte et Didelon 2011) d'autre part, ne permettent pourtant pas d'observer des logiques internes aux Etats, car les analyses se limitent au cadre international. De plus, ces publications s'en tiennent au seul couple présence/absence d'ambassades.

12 Je propose de l'aborder d'une manière différente ici en défendant le parti que la localisation des établissements à vocation diplomatique à l'intérieur d'un Etat ou d'une ville est également révélatrice de logiques spatiales entre la capitales et les grandes villes du pays. La seule modalité présence/absence d'ambassade résidente ne permet pas de prendre en compte tout le champ de la géographie diplomatique. J'ai joint à ce corpus la liste de tous les consulats présents en Allemagne en-dehors des sections consulaires des ambassades.

13 L'implantation de ces services diplomatiques (ambassades et consulats) a pu être recnstituée par pas de dix ans entre 1984 et 2014, ce qui permet de donner des éléments quant aux temporalités d'adaptation des appareils diplomatiques et consulaires à la nouvelle donne territoriale de la Réunification. Parmi les sources disponibles, on trouve à la fois les informations fournies par le site du Ministère fédéral des Affaires Etrangères pour l'année 2014, ainsi que celles de l'annuaire publié annuellement de la vie publique allemande, le Taschenbuch des öffentlichen Lebens Deutschland, pour les années 1984, 1994 et 2004. La liste diplomatique consultée dans les archives de l'ambassade de France à Berlin-Est fournit la liste des ambassades et consulats présents en RDA pour l'année 1986. Les consulats honoraires, dont l'activité se limite souvent à celle d'une personne privée, et qui ne se concrétisent pas par une véritable administration, capable de fournir un passeport par exemple, n'ont pas été retenus. Ils sont d'ailleurs souvent sous la tutelle de consulats ou de consulats généraux, qui ont une compétence territoriale souvent plus étendue et des bâtiments strictement dédiés aux questions administratives en lien avec le pays représenté.

Les modalités de présence de la diplomatie étrangère varient beaucoup d'un Etat à l'autre. L'Allemagne abrite 160 ambassades $^{1}$ en 2016 comme en 2014, dernière année de mon étude qui fait du pays, le $5^{\mathrm{e}}$ au monde pour la taille de l'appareil diplomatique accueilli. Berlin a donc la capacité d'attirer également les «diplomaties faibles » pour reprendre l'expression de Beauguitte et Didelon (2011) pour désigner les Etats qui n'envoient ni n'abritent beaucoup de représentations diplomatiques. Les autres Etats ceux qui ne sont pas présents par le biais d'une ambassade ou d'un consulat entretiennent des liens avec l'Allemagne via des structures situées dans d'autres capitales (en général Bruxelles ou Londres). On parle alors d' "ambassade non résidente ».

Si la majorité des Etats du monde ont en effet une ambassade en Allemagne, beaucoup moins ont des consulats. On retrouve pour ces derniers une structure très discriminante entre diplomaties faibles et fortes déjà étudiée pour les comparaisons 
entre les Etats, dans leur capacité à implanter un grand nombre d'ambassades dans des capitales étrangères. Le nombre d'Etats à posséder au moins 2 consulats ${ }^{2}$ est de 48 en 1984. Il augmente au début des années 1990 et se situe dans un intervalle allant de 66 (1994) à 72 (2014). On retrouve (figure 2) parmi ces Etats très présents à la fois les plus puissants mais surtout ceux ayant le plus de nationaux sur le sol allemand, qu'il s'agisse majoritairement d'expatriés travaillant pour des entreprises et de militaires (EtatsUnis, France, Royaume-Uni) ou de population immigrée de longue date (Turquie, Italie, Grèce). La proximité avec l'Allemagne peut également jouer à la marge, surtout dans les années 1980 à 1990, pour des Etats comme le Danemark ou la Suisse.

Figure 1 : Nombre d'ambassades et de consulats présents en Allemagne entre 1984 et 2014

\begin{tabular}{|l|c|c|c|c|c|}
\hline & $\begin{array}{c}1984 \\
\text { (RFA) }\end{array}$ & $\begin{array}{c}1986 \\
\text { (RDA) }\end{array}$ & 1994 & 2004 & 2014 \\
\hline Ambassades & 122 & 82 & 145 & 158 & 160 \\
\hline Consulats de compétence nationale & 60 & $?$ & 52 & 65 & 74 \\
\hline $\begin{array}{c}\text { Consulats et consulats généraux à } \\
\text { compétence régionale }\end{array}$ & 221 & 4 & 286 & 279 & 243 \\
\hline
\end{tabular}

Source : d'après Ministère fédéral des affaires étrangères allemand et Taschenbuch der öffentlichen Lebens Deutschland

Figure 2 : Etats avec au moins 7 consulats en Allemagne $^{3}$ entre 1984 et 2014

\begin{tabular}{|l|c|c|c|c|}
\hline & 1984 & 1994 & 2004 & 2014 \\
\hline Turquie & 14 & 15 & 13 & 13 \\
\hline Italie & 11 & 12 & 12 & 8 \\
\hline France & 12 & 10 & 7 & 7 \\
\hline Etats-Unis & 6 & 7 & 6 & 7 \\
\hline Grèce & 9 & 9 & 9 & 6 \\
\hline Espagne & 9 & 7 & 7 & 6 \\
\hline Yougoslavie/Serbie & 12 & 7 & 6 & 6 \\
\hline Suisse & 7 & 8 & 7 & 4 \\
\hline Danemark & 5 & 8 & 5 & 3 \\
\hline Royaume-Uni & 7 & 6 & 6 & 3 \\
\hline Suède & 7 & 4 & 2 & 1 \\
\hline
\end{tabular}

Source : d'après Ministère fédéral des affaires étrangères allemand et Taschenbuch der öffentlichen Lebens Deutschland

Le nombre d'ambassades augmente entre 1984 et 2014 sous l'effet de deux logiques conjointes (figure 1). La première tient de l'augmentation globale du nombre d'Etats dans les années 1990, en particulier suite aux dislocations de l'URSS et de la Yougoslavie. Deuxièmement, l'Allemagne a, sur la même période, vu croître son influence sur le plan diplomatique. Son importance dans les relations entre Etats européens ou entre Union Européenne et Etats tiers n'est plus à démontrer. De même, sur le plan économique, elle est devenue l'un des premiers partenaires de très nombreux Etats. Mécaniquement, elle devient donc un pays où même un Etat de taille modeste va chercher à installer une ambassade. En ce qui concerne les consulats, la logique est différente. Certes, leur nombre augmente comme les ambassades entre 1984 et 1994, du fait également de l'augmentation du nombre d'Etats à représenter. A titre 
d'exemple, en 1994, la Croatie a 5 consulats, l'Ukraine 4, la Bosnie-Herzégovine 3, autant de structures qui ne pouvaient exister avant 1990. De plus, certains Etats existant avant 1989 et ayant connu des transitions démocratiques dans le même temps ont aussi ouvert des consulats. La Pologne et la Hongrie, par exemple, en ouvrent 4 chacune. Le nombre de consulats diminue par la suite. Dans les années 2000 et 2010, le nombre de fermetures est important, en particulier dans les chancelleries d'Etat relativement riches, bien dotés en structures diplomatiques. Cela s'explique par la crise des finances publiques dans de nombreux Etats, en particulier européens, qui conduisent à la fermeture de structures coûteuses, notamment en termes de personnel et d'immobilier. De plus, après la généralisation de la fourniture en passeports biométriques après les attentats de 2001 aux Etats-Unis, les consulats ont besoin de moyens très importants pour procurer des documents d'identité et des visas. Leur coût de fonctionnement a augmenté, poussant de nombreux Etats à fermer des structures ou à nommer à leur place des consuls honoraires. Parallèlement, le panel de formalités dématérialisées s'est aussi beaucoup étendu, réduisant ainsi la nécessité du maintien de nombreuses infrastructures. La Suède passe ainsi de 7 consulats en 1984 à un seul, rattaché à son ambassade de Berlin en 2014. Dans la même période, le nombre de consulats français passe de 12 à 7 , dont certains sont maintenus officiellement mais ferment leur permanence comme à Hambourg en 2013. De même, si les Etats-Unis maintiennent sur la période un nombre constant de consulats, seuls trois sont désormais habilités à fournir des passeports.

17 Le nombre de consulats en Allemagne reflète les tensions de la géopolitique mondiale, la géographie de l'origine de la population étrangère dans le pays ainsi que le lent rapprochement de la première puissance économique d'Europe du rang des plus grandes diplomaties.

\section{Ambassades et consulats : le poids des capitales et la concentration dans les grandes villes}

18 L'Allemagne abrite un grand nombre de consulats et d'ambassades. Leur répartition sur le territoire demande d'être interrogé car il permet de mettre en évidence le poids respectif des capitales (Bonn, Berlin-Est puis Berlin unifié) et des grandes villes entre les années 1980 et 2010.

\section{A. Une géographie des ambassades très réactive à la Réunification}

La localisation des ambassades sur un territoire présente un caractère en général très monotone puisque rares sont les cas où la capitale d'Etat n'abrite pas l'intégralité des ambassades résidentes dans le pays. Comme le note Anne-Thida Norodom (2015), rares sont les cas de distorsion entre résidence du gouvernement et capitale diplomatique comme en Côte d'Ivoire entre Yamoussoukro et Abidjan. Ces exceptions sont d'ailleurs révélatrices de capitales officielles considérées comme fantoches, qui ne sont finalement pas celles où se trouve véritablement l'appareil d'Etat. Dans l'Allemagne divisée, c'est donc naturellement à Berlin-Est et à Bonn que se trouvaient les ambassades, ouvertes de manière progressive au gré des indépendances d'anciens Etats colonisés et des reconnaissances de la souveraineté des deux Etats allemands. On ne 
trouve que deux exceptions d'ambassades (la Pologne et l'Erythrée) situées à Cologne mais dont les relations, par ailleurs, avec la RFA étaient faibles.

En 1990, cette géographie des ambassades se modifie brutalement et s'adapte en quelques mois seulement à la disparition de la RDA. La Réunification conduit en effet à l'extension de l'aire de compétence du droit ouest-allemand et à la dissolution des institutions est-allemandes. Bonn devient le lieu unique d'exercice du pouvoir en Allemagne. Ce mouvement est facilité par le fait qu'il y avait beaucoup plus d'ambassades à Bonn qu'à Berlin-Est sans en avoir une également à Bonn. Aucun Etat dans les années 1980 ne possédait d'ambassade en RDA sans en avoir une également à Bonn. La question d'un déménagement à Berlin de la capitale fédérale n'est pas encore tranchée et même loin d'être arrêtée (elle ne l'a été qu'en juin 1991 après d'âpres débats), et le transfert de capitale s'inscrit, en l'absence de calendrier dans le long terme. Les chancelleries n'ont pas attendu les tergiversations allemandes sur un déménagement futur de leur capitale avant d'organiser leurs services. Il n'est pas donc pas étonnant qu'aucune ambassade ne soit restée à Berlin en 1990, même si quelques Etats y ont laissé des antennes ou ont transformé leurs locaux en consulats. C'est ainsi qu'alors que Berlin se trouvait vide d'ambassades, le nombre de représentations diplomatiques s'est non seulement maintenu à Bonn mais a même augmenté si on considère l'installation des représentations de nouveaux Etats au début des années 1990.

21 Une fois la décision de transférer la capitale votée, Bonn devient un lieu perçu comme provisoire. Les autorités allemandes parlent d'un déménagement pour 2000. Il aura lieu en 1999. La diplomatie s'avère être l'une de ces fonctions indirectes de capitale, qui demandent à être près du gouvernement sans que ce soit inscrit dans la loi, ni qu'aient été prévues dans le budget fédéral des dispositions financières pour la migration des institutions. Dans ce domaine, les chancelleries se comportent un peu comme des acteurs privés, avec une relative liberté de circulation et d'installation. Le premier effet de ce constat est, bien sûr, que tous les Etats n'ont pas les mêmes capacités quant à leur installation à Berlin et les conditions d'hébergement de leurs services. Là, il ne s'agit plus des conditions géopolitiques, mais de questions pratiques liées à la position dans l'espace d'un gouvernement qui impacte la géographie des représentations diplomatiques.

A partir de 1999, les différences entre Etats sont donc moins politiques qu'économiques. Dans l'ensemble, les Etats les plus riches, environ 80, dont les 15 membres d'alors de l'Union européenne et sa périphérie proche, la Russie, le Japon ou les Etats-Unis suivent directement le gouvernement allemand en partant à Berlin dès 1999. Ils sont rejoints à l'horizon 2001 par une trentaine d'Etats, en général émergents (Brésil, Chine, Egypte, Iran, Mexique). La quarantaine d'Etats restants partent à Berlin au cours de la décennie 2000, les plus lents à partir étant les plus pauvres. Les trois derniers ne déménagent leurs représentations de Bonn à Berlin qu'en 2010. L'idée d'une capitale bicéphale un temps imaginée, entre un Berlin très politique, et une ville de Bonn spécialisée dans l'accueil d'institutions chargées du développement, imaginée au début des années 2000, n'a pas été suivie. 


\section{B. Un système de villes finalement peu modifié par la Réunification}

La répartition des consulats en Allemagne met en évidence un système de villes qui apparaît comme multipolaire ou polycentrique. Les consulats sont des services de haut niveau, qu'aucun Etat au monde n'a les moyens d'implanter dans toutes les villes et qui par conséquent en privilégient un tout petit nombre (figure 3). Tout au long de la période, Bonn et Berlin accueillent à eux deux environ $40 \%$ des consulats. Les proportions du nombre de consulats dans l'une et l'autre ville s'inversent du fait du transfert des institutions fédérales. Tant que Bonn reste capitale effective, la ville concentre un grand nombre de consulats, qui décline doucement du fait du départ des ambassades (et donc des services consulaires qui vont avec) ce qui s'effectue progressivement. Dans un deuxième temps, des Etats vont aussi garder une antenne de leur ambassade à Bonn même si l'ambassadeur réside bien à Berlin depuis plusieurs années. Le poids de Berlin s'accentue dès les années 1990 avec l'ouverture de nombreux consulats mais ne devient vraiment très important qu'après le déménagement de la majorité des ambassades une dizaine d'années plus tard.

Figure 3 : Répartition des consulats entre les villes allemandes entre 1984 et 2014
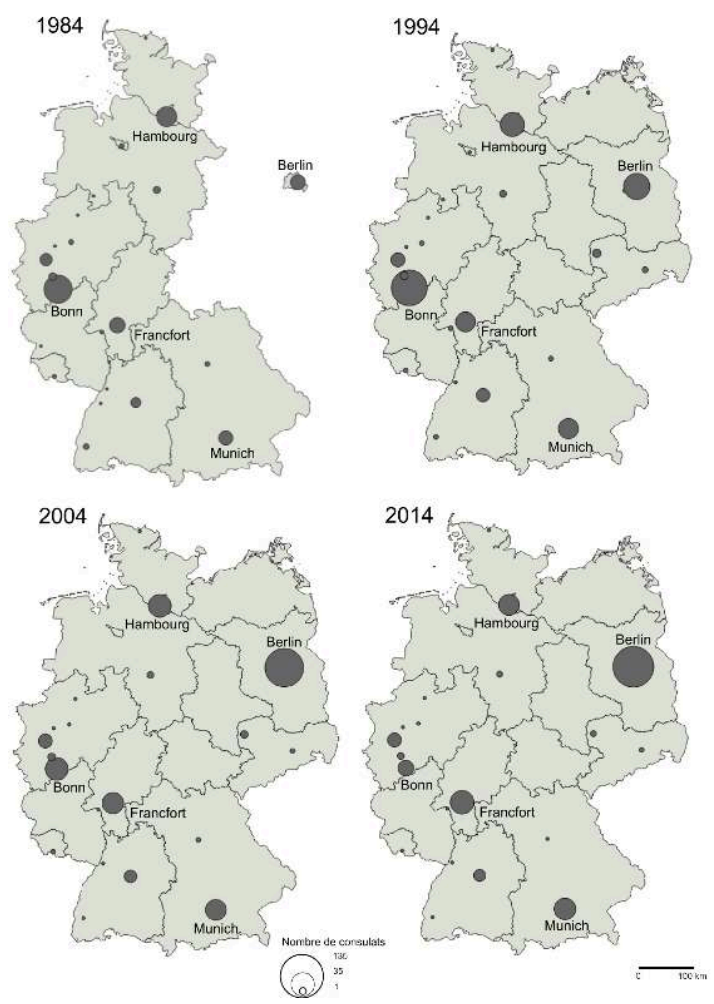

Source : d'après Ministère fédéral des affaires étrangères allemand et Taschenbuch der öffentlichen Lebens Deutschland

Les deux capitales, Hambourg, Francfort et Munich abritent ensemble ainsi entre 71 et $83 \%$ de l'ensemble des consulats. Ce pourcentage augmente à mesure que le nombre de consulats baisse dans les années 2000 et 2010. Le nombre d'Etats à posséder des consulats change peu; on compte plutôt moins de consulats par Etat représenté, d'où un resserrement sur ces quelques grandes villes et une baisse du poids des villes à la position qu'on pourrait qualifier d'intermédiaire, comme Fribourg ou Hanovre. La part 
importante des consulats situés dans ces cinq villes confirme l'hypothèse de clusters diplomatiques ou consulaires, dans des agglomérations qui ont une tradition ancienne de lieu de diplomatie comme Munich, lieu de résidence de la Cour de Bavière jusqu'à la Première Guerre mondiale, ou Hambourg, ville libre et quasi souveraine jusqu'à la fin $\mathrm{du} \mathrm{XIX}^{\mathrm{e}}$ siècle. La présence de ports maritimes (Hambourg) ou d'institutions internationales (Bonn, Francfort, Munich) joue également un rôle important, bien que difficilement quantifiable dans la présence de consulats. Le rôle des autres villes n'est pas marginal et concerne des Etats ayant un grand nombre de consulats du fait d'une population expatriée nombreuse (comme les consulats turcs auprès des immigrés résidant dans la Ruhr à Essen et Dortmund). Quelques villes frontalières ont aussi tendance, malgré leur faible taille, à accueillir un consulat de l'Etat voisin comme Fribourg pour la Suisse ou Flensbourg pour le Danemark.

Autre enseignement, on ne constate pas d'installation massive de consulats dans les villes d'ex-RDA (hors Berlin) après la Réunification, en tous les cas beaucoup moins que leur taille ne le laisserait supposer. En 1994, Leipzig, Dresde et Rostock rassemblent au total 3\% de l'ensemble des consulats. Cette proportion est aujourd'hui réduite de moitié. Cela s'explique en partie par la proximité de Berlin, où de nombreux Etats installent des consulats dès le début des années 1990 et dont la circonscription consulaire inclut l'intégralité du territoire précédemment est-allemand. A la différence du système urbain de l'ancienne Allemagne de l'Ouest, en ex-RDA, la capitale n'a pas de concurrente directe en termes de taille et de richesse, et son influence se confond beaucoup plus avec les limites de son Etat. C'est en partie une conséquence, mais il n'y a pas non plus l'héritage, dans ces villes d'ex-RDA extérieures à Berlin, d'un appareil consulaire important qui daterait de la RDA. En 1986, on ne compte que 4 consulats en dehors de Berlin-Est, 2 à Leipzig et 2 à Rostock. Malgré le maintien de ces structures après 1990, leur faible effectif ne permet absolument pas d'effet d'entraînement, d'où la rareté d'ouverture de consulats à Leipzig et à Dresde. Dans ce domaine comme dans la répartition des sièges des plus grandes entreprises allemandes notamment, les villes des régions orientales (Berlin y compris parfois) apparaissent déclassées par rapport aux villes des régions occidentales, qui ont maintenu leur niveau de service tel qu'il était dans les années 1980 .

Les consulats, en tant que « services rares » se situent de plus en plus dans les grandes villes à mesure que leur nombre diminue, selon un principe bien connu dans les modèles de répartition des services. L'implantation des consulats définit cependant également des circonscriptions et donc une aire géographique dont on dépend. Le définition de cette dernière perpétue-t-elle, à l'aune de la raréfaction de ces structures l'ancienne frontière inter-allemande?

\section{Les circonscriptions consulaires et l'ancienne frontière interallemande}

27 A la manière d'un tribunal ou d'une préfecture, les consulats (ou les sections consulaires des ambassades), en tant que service public, ont donc une aire de compétence claire. Cependant, les circonscriptions consulaires sont définies par des autorités indépendantes de l'Allemagne. Elles n'ont aucune obligation de se mouler dans la structure administrative du pays. La pratique montre d'ailleurs qu'aucun Etat ne reprend exactement la structure des Länder en premier lieu parce qu'aucun d'entre 
eux n'a autant de consulats en Allemagne qu'il n'y a de Länder. C'est pourquoi les circonscriptions consulaires ont tendance le plus souvent à les regrouper. Il arrive aussi également que les Etats en viennent à diviser les Etats-régionaux en plusieurs circonscriptions consulaires, lorsqu'ils paraissent trop grands et trop peuplés. Les choix opérés en termes de découpage répondent à un certain nombre de logiques dont on peut aisément tirer des hypothèses. La première est que ces circonscriptions vont regrouper des ensembles homogènes en termes de population, notamment de la population étrangère représentée. Par exemple, jusque dans les années 1990, il ne serait pas étonnant de noter une concentration plus forte, comparativement à la population résidente, de consulats français dans les Länder bordant la frontière avec la France du fait de la présence de nombreux militaires français résidant dans ces mêmes régions. Une deuxième serait que les circonscriptions consulaires sont créées sous la forme d'ensembles polarisés par une grande ville concentrant un certain nombre de consulats.

Une troisième, à laquelle nous attacherons une attention particulière, serait le maintien, pour des raisons fonctionnelles ou historiques, de la division entre ex-RDA et l'ancien périmètre fédéral dans le découpage des circonscriptions comme il l'est dans le découpage des Länder (Berlin excepté).

Figure 4 : Typologies présentant les liens préférentiels des régions allemandes avec les villes où sont situés les consulats compétents

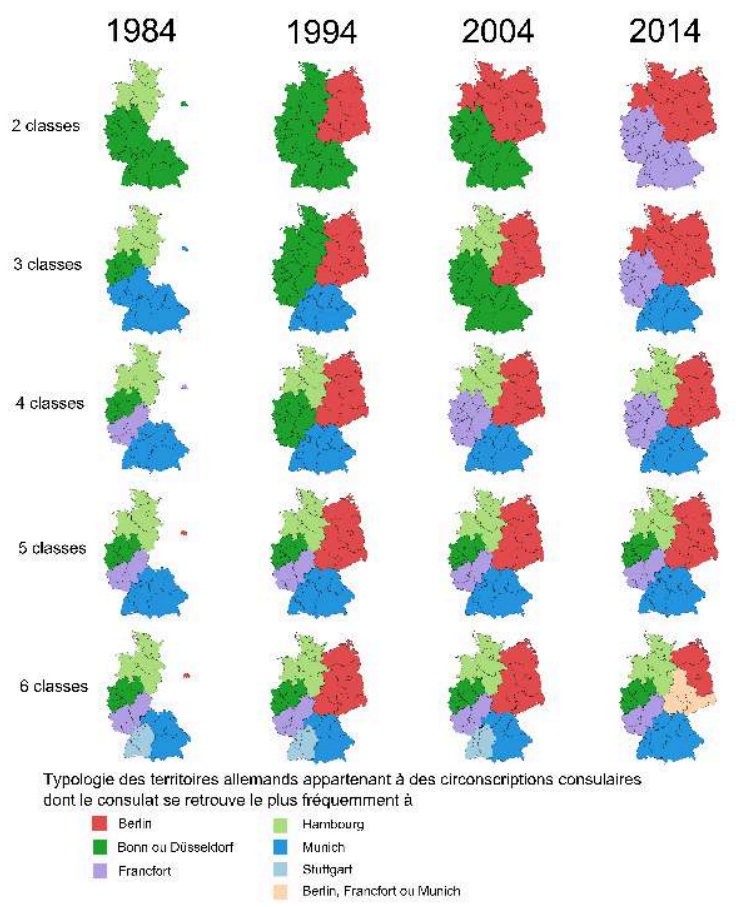

Source : Taschenbuch des öffentlichen Lebens et Auswärtiges Amt. Calculs de l'auteur

Pour étudier cette question, j'ai reconstitué les circonscriptions consulaires dessinées par les Etats représentés possédant au moins deux consulats, donc obligés de diviser au moins en deux le territoire allemand. Pour certains Etats qui ont plusieurs consulats en Allemagne, celui de la capitale est compétent pour l'ensemble du pays, ce qui implique 
que des régions soient couvertes deux fois, par le consulat bonnois ou berlinois et par un consulat situé ailleurs. Dans ces cas-là, nous avons retenu comme circonscriptions consulaires l'ensemble des régions exclusivement couvertes par la capitale. Nous avons opéré des Classifications Ascendantes Hiérarchiques sur ce corpus avec pour variables les villes dans lesquelles se trouvent les consulats compétents, et pour éléments les entités qui n'étaient découpées par aucune circonscription consulaire, formant 34 régions souvent plus petites que les Länder (figure 4).

Le premier enseignement de ces typologies tient dans la robustesse du découpage de l'espace allemand en Länder. Les Etats ayant placé les parties d'un même Land sous l'autorité d'un même consulat s'avèrent peu nombreux et le sont de moins en moins au cours de la période. Aucune typologie avec 6 classes ne fait apparaittre de polarisations différentes entre plusieurs parties d'un même Land. Le deuxième est également la solidité de moins en moins visible de l'ex-RDA comme périmètre de circonscriptions consulaires. En 1994, seules 7,4 \% des circonscriptions dessinent des territoires regroupant des régions des deux anciennes Allemagnes, circonscriptions que nous qualifierons de "mixtes ». Ce pourcentage monte à $22 \%$ en 2004 et atteint en 2014 $28,9 \%$. Si on ajoute à ces mesures les circonscriptions uniques pour le territoire allemand, cette proportion s'élève pour 1994, 2004 et 2014 à respectivement $24 \%, 36 \%$ et $45 \%$.

Figure 5 : Evolution du périmètre de circonscriptions consulaires situées à Berlin, Bonn, Francfort et Munich

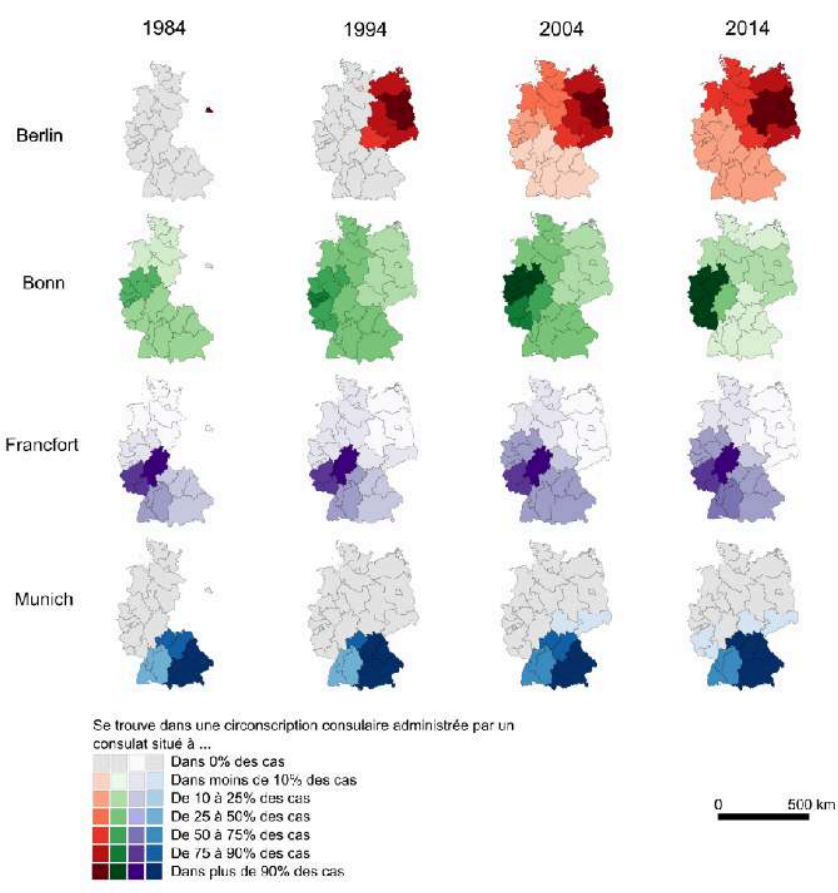

Source: Taschenbuch des öffentlichen Lebens et Auswärtiges Amt. Calculs de l'auteur 
fermer un dans une ville secondaire des régions occidentales. La France, par exemple, étend la circonscription de son consulat de Berlin, ouvre un consulat à Leipzig compétent pour la Saxe et la Thuringe, et ferme en retour ses consulats de Fribourg et de Baden-Baden. Lorsque l'ex-RDA se trouve sous la juridiction de plusieurs consulats, comme nous l'avons vu plus haut, il est finalement rare que ce soit du fait d'ouvertures de consulats à Leipzig et à Dresde mais plutôt la constitution de circonscriptions consulaires mixtes depuis Hambourg (comprenant le Mecklembourg-Poméranie Occidentale) ou Francfort (comprenant la Thuringe). Ces tendances sont timides au début de la période et tendent à se renforcer. 20 ans plus tard, seuls 13 Etats conservent des circonscriptions depuis Berlin recoupant exactement l'ancienne RDA. Ces tendances sont également visibles lorsqu'on étudie la compilation des circonscriptions consulaires ville par ville (figure 5). Berlin voit son influence sortir de son territoire municipal dans les années 1980 à des circonscriptions consulaires souvent étendues audelà de son environnement proche alors que Bonn, que la position de capitale destinait à des circonscriptions consulaires étendues, voit son rôle se renforcer sur sa région. Dans le même temps, Francfort et Munich, à l'ancrage local plus affirmé voient leurs circonscriptions consulaires s'étendre au fil du temps, la première moins timidement que la seconde.

L'année 2014 est marquée par la réduction très visible du nombre de consulats par Etat. Pour nombre de chancelleries, le recentrage sur la partie ouest du pays se fait surtout sur Francfort, qui a récupéré des consulats situés jusque-là à Bonn ou à Düsseldorf, avec des circonscriptions consulaires souvent supérieures à 30 millions d'habitants. L'exRDA, plus que de former un territoire cohérent se trouvant recouper l'aire d'influence de Berlin et ses marges, devient le cœur d'un espace s'étendant aux dépens de Hanovre, Dresde et Leipzig dans un premier temps et de plus en plus Hambourg. C'est pourquoi se renforce la fusion des Länder de l'est et du nord du pays dans des mêmes entités, augmentant ainsi le nombre de circonscriptions consulaires mixtes.

\section{Conclusion}

33 A mesure que le maillage tissé par les consulats sur le territoire allemand se développe puis se rétracte dans les dernières années, on assiste indubitablement à un resserrement des fonctions diplomatiques sur quelques grandes villes. Berlin et Francfort s'avèrent les centres consulaires les plus importants, l'un parce que sa fonction de capitale lui permet de capitaliser sur les toujours plus nombreux Etats qui optent pour un réseau minimal de consulats, et le deuxième par une capacité à polariser une très large région sud et ouest de l'Allemagne, sans concurrence sérieuse dans la partie rhénane. Hambourg et Munich complètent ce tableau des villes d'abriter de nombreux consulats. Les circonscriptions consulaires, devenues souvent très grandes, ont bien souvent fait disparaître la frontière interallemande, pourtant encore très visible dans les découpages opérés dans les années 1990. Bien sûr, encore une fois, les consulats sont loin d'être les services publics à compter le plus d'usagers et la portée fonctionnelle de leur localisation a des effets socio-économiques marginaux. Néanmoins, le fait qu'en 2014, près de la moitié des circonscriptions consulaires regroupe des espaces situés autrefois des deux côtés du rideau de fer semble signifiant. Il révèle une perception nouvelle du territoire allemand sur un redécoupage des aires d'influence des grandes villes de manière de plus en plus indépendante de l'ancienne 
frontière. D'autres exemples, non plus le fait d'acteurs étrangers mais nationaux, vont dans le même sens comme la répartition des groupes publics de radio-télévision (la NDR de Hambourg, dans l'ancien périmètre fédéral, repose sur des Etats-régions anciens comme sur le Mecklembourg-Poméranie Occidentale, auparavant situé en RDA). Les quelques projets de fusion de Länder lient fréquemment anciens et nouveaux Etats-régions (Laporte, 2016b). Certes, l'approche de la frontière est ici purement administrative et ne tient pas compte des pratiques et des représentations individuelles. Elle ne répond pas non plus pour le moment à la question de la capacité de mouvements des découpages à avoir un impact sur les inégalités sociales et économiques. En l'état, elles semblent plus contribuer, au-delà d'une division est-ouest à creuser les écarts entre la tête du réseau urbain et le reste du territoire.

\section{BIBLIOGRAPHY}

Barakat B., 2015, «A "Recipe for Depopulation"? School Closures and Local Population Decline in Saxony ", Population, space and place, Vol. 21, p.735-753.

Beauguitte L., Didelon C., 2011, « Structures spatiales des relations diplomatiques mondiales », Dixièmes Rencontres de Théo Quant.

Boichot C., 2012, Centralités et territorialités artistiques dans la structuration des espaces urbains. Le cas de Paris et de Berlin, Thèse de doctorat à l'Université Paris 1 et Université Viadrina de Francfort sur l'Oder, $480 \mathrm{p}$.

charlot 0., 2008, « Après la frontière interallemande ", Trajectoires, Vol. 2, p. 9-20.

Cochrane A., Passmore A., 2001, « Building a national capital in an age of globalization: the case of Berlin », Area, Vol. 33, n 4, p.341-352.

commission Européenne, 2014, Sixième rapport sur la cohésion. Investissement dans l'emploi et la croissance. La promotion de l'emploi et de la bonne gouvernance dans les régions et villes de l'UE, Bruxelles, Commission européenne, 335 p.

Eurostat, 2015, PIB au niveau régional, disponible en ligne : http://ec.europa.eu/eurostat/ statistics-explained/index.php/GDP_at_regional_level/fr , 2015.

Florentin D., 2015, «La vulnérabilité des objets lents: les réseaux d'eau », Annales de la Recherche Urbaine, Vol. 110, p. 152-163.

Grésillon B., 2002, Berlin, métropole culturelle, Paris, Belin, 350 p.

Hentschker C., Mennicken R. et schmid A., 2014, « Defining hospital markets - an application to the Geman hospital sector », Health Economics Review, vol. 4, nº 28.

Hirschhausen B. von, Lacquement G. (dir.), 2007, Le développement rural en Allemagne réunifiée modèles, contextes, enjeux, Paris, Armand Colin, 207 p. 
Hirschhausen B. von, Roth H., 2003, « Les systèmes passent, les murs restent. Du rôle de l'immobilier d'entreprise dans la trajectoire du système industriel est-allemand ", Géocarrefour, Vol. 78, n 4, p. 349-362.

Huning S., Naumann M., Bens O. et Hüttl R. F., 2011, « Infrastructure Planning in Rural Regions: The Case of Water Infrastructures in Brandenburg, Germany ", European Planning Studies, Vol. 19, $\mathrm{n}^{\circ} 8$, p. $1499-1516$.

Krätke S., 2002, Medienstadt. Urbane Cluster und globale Zentren der Kulturproduktion, Opladen, Leske + Budrich, $267 \mathrm{p}$

Kühn M., 2014, « Peripheralization: Theoretical Concepts Explaining Socio-Spatial Inequalities », European Planning Studies, Vol. 23, n² 2, p. 367-378.

Lacquement G., Born, K. M., von Hirschhausen B., 2012, Réinventer les campagnes en Allemagne paysages, patrimoine et développement rural, Lyon, ENS éditions, 279p.

Laporte A., 2014, «Allemagne, 25 ans après la chute du Mur de Berlin : que reste-t-il de la ligne Est-Ouest? », Géoconfluences

Laporte A., 2016a, De Bonn à Berlin. Le transfert d'une capitale (1990-2010), Toulouse, Presses Universitaires du Midi, $300 \mathrm{p}$.

Laporte A., 2016b, «L'importance grandissante de Berlin en Allemagne comme conséquence de la rétraction des services publics de haut niveau ", Bulletin de l'Association des Géographes Français, Vol $\mathrm{n}^{\circ} 93, \mathrm{n}^{\circ} 1, \mathrm{p} .62-76$.

Maccarone O., sbordone L., 2003, « Berlino, città globale? », Bollettino della Società Geographica Italiana, Vol. $12, \mathrm{n}^{\circ} 8, \mathrm{p} .561-579$.

Mamadouh V., Meijer, A., Sidaway, J.D., Van der Wusten, H. 2015, « Toward an Urban Geography of Diplomacy: Lessons from The Hague ", The Professional Geographer, Vol. 67, nº 4, p. 564-574.

Manzagol Claude, 1991, « Les activités spécifiques » in Bailly A., Ferras R., Pumain D. (dir.), Encyclopédie de la géographie, Paris, Economica, pp. 489-514

Norodom Anne-Thida, 2015, « Capitale et relations diplomatiques : une pratique disparate » dans Laetitia Janicot, Olivier Renaudie et Franck Laffaille(dir.), Les villes capitales, miroirs de l'Etat ?, Pontoise, Université de Pontoise, p. 65 - 74.

Queva C., 2007, « Les Paradoxes de la Région en Allemagne, entre réseaux et territoires: la région, outil de déterritorialisation ", Annales de Géographie, nº653, p. 41-64.

Riquet P., 1996, « Europe médiane » in R. Brunet (dir.) Géographie universelle, Montpellier, GIPReclus, p. $234-462$.

Roth H., 2008, "Dynamiques industrielles et mutations des espaces ruraux en Allemagne ", Géocarrefour, Vol. 83, nº 4, p. 285 - 293.

sur E., 1997, « Pouvoir et territoire : le cas des Länder de la République Fédérale d'Allemagne », Bulletin de l'Association des Géographes Français, 1997, nº 2, p. 146-154.

\section{NOTES}

1. Parmi lesquels nous comptons les représentations des Territoires Palestiniens et de Taïwan, dont la souveraineté est contestée et pour lesquels le titre d'ambassade est encore impropre à Berlin en 2014. 
2. Avec donc au moins un consulat installé en-dehors de l'ambassade. Lorsque les Etats n'ont qu'un seul consulat, ce dernier est le plus souvent sis dans l'ambassade et sa circonscription consulaire se confond avec le territoire entier qui l'abrite.

3. Pour l'année 1984, le tableau ne prend en compte que la République fédérale.

\section{ABSTRACTS}

With 160 embassies and 317 consulates, Germany appears as one of the main nodes of diplomacy presence in the world. The unification and then the transfer of the institutions deeply modified the location of these consulates and embassies within the country. The study of this moving geography on 30-year period make possible to illustrate the transition between two sealed diplomacy systems when Germany was divided to a network of embassies and consulates affected by exogenous factors (progressive closing of these structures) and by endogenous factors. Concerning the last ones, we will focus on the hypothesis of a progressive erasing of the former German-German border in the way the city networks integrate the extension of the country to the so-called "new states" and the description of the extension of the consular districts.

Avec 160 ambassades et 317 consulats sur son sol en 2014, l'Allemagne apparaît comme un des plus importants nœuds au monde en termes de présence diplomatique. La Réunification puis le déplacement des institutions de Bonn à Berlin ont profondément modifié la répartition de ces établissements au sein de son territoire. L'étude de cette géographie mouvante sur une période de 30 ans permet de présenter le passage de deux systèmes hermétiques lorsque l'Allemagne était divisée, à un réseau d'ambassades et de consulats unique à la fois traversé par des logiques exogènes (la fermeture progressive de ces structures par de nombreuses chancelleries) et endogènes. En ce qui concerne ces dernières, nous nous intéresserons particulièrement à l'hypothèse d'un effacement progressif de l'ancienne frontière interallemande, autant dans la manière dont le réseau des villes abritant les consulats intègre les nouveaux Länder que dans la description des périmètres de compétence de ces institutions diplomatiques.

Mit 160 Botschaften und 317 Konsulate im Jahre 2014 sieht Deutschland als ein von den wichtigsten Ländern in der Welt für die diplomatische Anwesenheit. Die Wiedervereinigung und der Umzug der Institutionen von Bonn nach Berlin haben die Verteilung dieser diplomatische Dienst sehr geändert. Die bewegende Geogrpaphie auf einer 30-Jahre Periode zeigt die Transition zwischen zwei verschlossene diplomatische Systeme als Deutschland geteilt wurde und ein heutiges Netzwerk von Botschaften und Konsulate, das von sowohl endogen Faktoren (wie die Konsulateschließungen) als auch exogene Faktoren beeinflusst wurde. Wir werden die Hypothese einer progressive Verwischung der ehemalige deutsch-deutsche Grenze betonen und studieren, wie das Stadtnetzwerk mit der Integration der neuen Länder zu der BRD reagiert hat und mit der geographische Beschreibung der Konsularbezirke. 
INDEX

Mots-clés: Allemagne, ambassade, consulat, découpages, frontière interallemande, géographie de la diplomatie, service public

Schlüsselwörter: Botschaft, deutsch-deutsche Grenze, Deutschland, Geographie der Diplomatie, Konsulat, öffentlicher Dienst, territoriale Gliederung

Keywords: consulate, diplomacy geography, embassy, geographical breakdown, German-German border, Germany, public service

\section{AUTHOR}

\section{ANTOINE LAPORTE}

Maître de conférences ENS de Lyon, Univ Lyon, CNRS, ENS de Lyon, EVS, UMR5600, F-69007, France, 15 parvis René Descartes, BP 7000, 69342 Lyon cedex 07, antoine.laporte@ens-lyon.fr 